\title{
Interventions for treating children and adolescents with overweight and obesity: an overview of Cochrane reviews
}

\author{
Louisa J. Ells ${ }^{1} \cdot$ Karen Rees $^{2} \cdot$ Tamara Brown $^{1} \cdot$ Emma Mead $^{1,3} \cdot$ Lena Al-Khudairy $^{2} \cdot$ Liane Azevedo $^{1}$. \\ Grant J. McGeechan $\mathbb{1}^{1} \cdot$ Louise Baur $^{4} \cdot$ Emma Loveman $^{5}$ - Heather Clements ${ }^{1}$ - Pura Rayco-Solon ${ }^{6}$. \\ Nathalie Farpour-Lambert ${ }^{7} \cdot$ Alessandro Demaio $^{6}$
}

Received: 19 September 2017 / Revised: 17 May 2018 / Accepted: 15 June 2018 / Published online: 9 October 2018

(c) The Author(s) 2018. This article is published with open access

\begin{abstract}
Children and adolescents with overweight and obesity are a global health concern. This is an integrative overview of six Cochrane systematic reviews, providing an up-to-date synthesis of the evidence examining interventions for the treatment of children and adolescents with overweight or obesity. The data extraction and quality assessments for each review were conducted by one author and checked by a second. The six high quality reviews provide evidence on the effectiveness of behaviour changing interventions conducted in children $<6$ years ( 7 trials), 6-11 years (70 trials), adolescents $12-17$ years (44 trials) and interventions that target only parents of children aged 5-11 years (20 trials); in addition to interventions examining surgery $(1$ trial $)$ and drugs $(21$ trials). Most of the evidence was derived from high-income countries and published in the last two decades. Collectively, the evidence suggests that multi-component behaviour changing interventions may be beneficial in achieving small reductions in body weight status in children of all ages, with low adverse event occurrence were reported. More research is required to understand which specific intervention components are most effective and in whom, and how best to maintain intervention effects. Evidence from surgical and drug interventions was too limited to make inferences about use and safety, and adverse events were a serious consideration.
\end{abstract}

Electronic supplementary material The online version of this article (https://doi.org/10.1038/s41366-018-0230-y) contains supplementary material, which is available to authorized users.

Louisa J. Ells

L.Ells@tees.ac.uk

1 School of Health and Social Care, Teesside University, Middlesbrough, UK

2 Division of Health Sciences, University of Warwick, Coventry, UK

3 University of Nottingham, Nottingham, UK

4 University of Sydney, Sydney, NSW, Australia

5 Effective Evidence LLP, Hampshire, UK

6 World Health Organization, Geneva, Switzerland

7 Obesity Prevention and Care Program, University Hospitals of Geneva, Geneva, Switzerland

\section{Introduction}

Excess weight in children and adolescents is a growing public health crisis [1-3], with inequalities occurring in populations from different socioeconomic [4-6] and ethnic groups [7-10].

Children and adolescents with obesity can develop a number of serious related comorbidities. These include musculoskeletal conditions [11], cardiovascular risk factors such as hypertension, insulin resistance, and hyperlipidaemia [12], respiratory conditions, including sleep apnoea [13] or asthma [14], and digestive diseases such as nonalcoholic fatty liver disease [15]. Psychosocial well-being can also be affected, with young people with obesity more susceptible to stigmatisation [16], reduced self-esteem and quality of life [17]. Evidence also demonstrates that obesity in childhood tracks into adulthood [18, 19], thus increasing the risk of ill health later in life [20, 21].

Given the complex nature of obesity, there is unlikely to ever be one treatment regime that will be effective across all populations, with the most suitable intervention approach determined by the child's age and degree of excess weight, 
amongst other considerations. Treatment options range from lifestyle modification interventions, to the use of bariatric surgery and drugs.

The least invasive and most widely used approach to treating obesity in childhood is lifestyle modification. These programmes aim to improve dietary quality, increase physical activity levels and reduce sedentary behaviours, often incorporating behaviour changing techniques to help sustain positive changes and prevent relapse. Many interventions have a family focus, with parents defined as the "agents for change", particularly in children under 12 years [22].

Forms of bariatric surgery include gastric bypass, sleeve gastrectomy and gastric banding [23]. Drugs used to treat obesity include: Sibutramine an appetite suppressant that, while still licensed in Brazil, was suspended by the European Medicine Agency and withdrawn by the Food and Drug Administration (FDA) in 2010 due to adverse cardiovascular effects; Orlistat, a fat absorption inhibitor that has been approved by the FDA but only for children $\geq 12$ years old [24]. Other drugs frequently used off license to treat obesity in childhood include: metformin, an anti-diabetic medication [25, 26] and fluoxetine, an antidepressant [27]. New drugs targeting appetite regulation are currently under development or evaluation.

The aim of this review was to conduct an integrative overview of six Cochrane reviews [28-33], to provide a comprehensive update to the previous Cochrane review on interventions for treating obesity in children [34].

This overview was written to help inform ongoing work by the World Health Organization on the management of children and adolescents with overweight and obesity.

\section{Methods}

We conducted this overview of reviews in accordance with the recommendations for Cochrane overviews of reviews [35]. PROSPERO (CRD42016053423). All reviews produced to update the Oude Luttikhuis (2009) review [34] were included.

A detailed description of the methods can be found in the online supplementary appendix 1 . In brief, data was extracted using a standardised data collection form, and review quality was assessed using the revised Assessment of Multiple Systematic Reviews (R-AMSTAR) measurement tool [36], by one reviewer and checked for accuracy by a second reviewer, with disagreements resolved by consensus. Each R-AMSTAR assessment was conducted by reviewers who were not authors of the original review.

The primary outcomes of interest were changes in BMI or BMI $z$-score, with results from relevant meta-analyses extracted alongside secondary outcomes as reported in the summary of findings tables. The original review authors'
Cochrane 'risk of bias' assessment [37] and Grading of Recommendations Assessment, Development and Evaluation (GRADE) assessment were also extracted.

\section{Results}

\section{Characteristics of included studies}

The characteristics of the six included reviews and the Randomised Controlled Trials (RCTs) included in each review are shown in the appendix: Supplementary Tables S1 and S2 (online Supplementary file), respectively. All six reviews were published between 2015 and 2017, and included RCTs with a minimum of six months data from baseline. Across all reviews a total of 163 studies (19,756 participants) were included, representing trials conducted between 1968 and 2016, from 30 countries. The vast majority of studies were undertaken in the USA $(n=73$, $45 \%)$ or Europe $(n=44,27 \%)$, with only $16(10 \%)$ studies conducted in upper-middle-income countries, while the remainder were conducted in high-income countries. (According to the World Bank list of economies (July 2016) http://siteresources.worldbank.org/DATASTATISTICS/

Resources/CLASS.XLS) Most studies were published within the last two decades.

The number of trials included in each review varied substantially from just one trial included in the surgery review [28], to 70 trials included in the review of lifestyle interventions in children aged 6-11 years [32]. Most trials were individually randomised, with a small number of cluster trials $(n=11)$ across all six reviews. The median sample sizes for included studies within each review ranged from 50 to 96, with individual trial samples sizes within reviews ranging from just 10-686 participants. Participant views (e.g., satisfaction with, or opinions of the intervention) were not reported in any of the surgery or drug trials, and were only reported in 23 trials across the four lifestyle reviews.

\section{Participants}

Every review excluded children who were critically ill or diagnosed with a syndromic form of obesity. Where applicable, pregnant and breast feeding females were also excluded. All reviews included children with obesity, and the four lifestyle intervention reviews also examined children with overweight (median BMI $z$-score across the lifestyle reviews was between 2.2 and 2.3, although calculated using a variety of different growth references). The surgery and drug reviews included any child under the age of 18 years. A priori mean age groups were set for trials included in the lifestyle reviews to ensure each trial was 
only included in one review (the lifestyle interventions targeting the child and parent, or child alone were reviewed in the following age groups: up to 6, 6-11 and 12-17 years; for lifestyle interventions targeting the parent as the sole agent for change, an age range of 5-11 years was included). The median proportion of females in each review ranged from 54 to $65 \%$, and reporting of socioeconomic status and ethnicity was limited.

\section{Interventions}

All reviews in this series examined the effectiveness of interventions that aimed to treat children or adolescents with overweight or obesity. One review [28] examined the effectiveness of bariatric surgery, another [29] studied drug interventions, whilst the remaining reviews [30-33] examined the effectiveness of lifestyle interventions that delivered diet, physical activity and behavioural interventions either as a single or multicomponent programme. Of these, Loveman (2015) [30] focused on parent-only interventions whilst Colquitt (2016) [31], Mead (2017) [32] and AlKhudairy (2017) [33] examined any lifestyle intervention by age group of the participating child. Interventions could be undertaken in any setting, although more than half of the trials $(n=85)$ were undertaken in either primary or secondary care.

\section{Comparators}

Comparators in each of the six reviews included true control (placebo or no intervention) $(n=38)$, usual care (defined by either the study author or reviewer) $(n=72)$ or an alternative concomitant therapy providing it was delivered in both the intervention and comparator arms $(n=53)$.

\section{Outcome measures}

All six reviews examined the same primary outcome measures (BMI / BMI $z$-score, body weight and adverse events) and secondary outcome measures (health-related quality of life; self-esteem; all-cause mortality; morbidity; body fat distribution; behaviour change; participants' views of the intervention; socioeconomic effects).

\section{Methodological quality of included reviews}

The R-AMSTAR assessment results for each review are shown in supplementary appendix Table S3. All six reviews scored between 35 and 41, out of a possible 44, and were therefore deemed of good methodological quality. Areas where all reviews were marked down included not providing a clinical consensus statement and not adequately describing statistical tests.

\section{Risk of bias of included randomised controlled trials}

The bias associated with the included trials varied across the six reviews (Appendix Table S4, supplementary file). In general random sequence generation was rated as low risk of bias for the majority of included studies. Allocation concealment was generally rated low or unclear risk of bias. Performance bias (i.e. not blinding study participants and personnel) was rated as high or unclear risk for the majority of the non-drug trials. Detection bias (i.e. not blinding outcome assessment) varied across the reviews, with lower risk of bias for objective outcomes (which included body mass measurements). Attrition bias (Attrition bias was determined by assessing the completeness of the outcome data, including attrition and exclusions from the analysis.) (i.e., incomplete data), was rated as unclear or high risk, in over half of all trials included in each review. Selective reporting bias (i.e., differences between reported and unreported findings) was generally poor in a large proportion of trials in each review. The proportion of trials with low risk of other biases varied across the reviews.

\section{Quality of evidence from randomised controlled trials in the included reviews}

Each of the six reviews assessed overall quality of the evidence using the GRADE method which is shown in the summary of findings (Supplementary appendix: Tables S5A-E,). Overall, the quality of the evidence was low for BMI and, where provided, was very low or low for other outcomes measured in the reviews. No studies provided data on socioeconomic effects. Reasons for downgrading BMI evidence included: high risk of bias (e.g., attrition), imprecision (wide confidence intervals), and inconsistency (heterogeneity).

\section{Effects of interventions}

Summary of findings (Supplementary appendix: Tables S5A-E), and BMI and BMI $z$-score outcome analyses (Supplementary appendix: Table S6) are presenting in the supplementary file.

\section{Lifestyle interventions for the treatment of overweight or obesity}

The vast majority of evidence (141 out of 163 trials) reviewed in this overview were lifestyle interventions (i.e. those that addressed diet, physical activity and / or behaviour change). This evidence was assessed across four reviews, examining the effectiveness of interventions delivered to the child, or parent and child across infancy, pre adolescence and adolescence. This was supplemented 
by a further review that specifically examined interventions that targeted parents as the sole agent for change in their child. The results are summarised below.

\section{Interventions for pre-school children up to the age of 6 years}

Colquitt et al. (2016) [31] conducted searches up to March 2015 and identified seven completed and four ongoing trials. Of the seven completed trials (923 participants), six tested multicomponent interventions and one tested a dietary intervention. Trials were undertaken in four countries (one upper-middle income) in a variety of settings, and all were published from 2009. The mean age of participants ranged from 2 to 5 years, and the median of the mean baseline BMI $z$-score was 2.25 . The proportion of female and white participants ranged from 25 to $80 \%$ and 47 to $91 \%$, respectively. The duration and nature of the intervention and comparators varied across the studies, and whilst all seven trials reported a period of post intervention follow-up ranging in duration from 6 to 32 months, followup data were only available for five studies.

When the multicomponent interventions were compared with control (usual care, enhanced usual care, or information provision) at the end of the intervention (6 to 12 months), a reduction in BMI $z$-score was observed in favour of the intervention: mean difference (MD) -0.3 units (95\% CI: -0.4 to -0.2 ) (210 participants; four trials). This reduction was maintained at both 12 to 18 months follow-up from baseline (6-8 months post intervention) (MD -0.4 units (95\% CI: -0.6 to -0.2 ); 202 participants; four trials), and at 2 years follow-up from baseline (12 months post intervention) (MD -0.3 units (95\% CI: -0.4 to -0.1 ); 96 participants; one trial). Three out of the four multicomponent studies also assessed parental weight change (parents were required to have a BMI of least 25 or $27 \mathrm{~kg} / \mathrm{m}^{2}$ to be included). Results from the parental weight change analysis revealed an overall mean difference of $-4.69 \mathrm{~kg}(95 \% \mathrm{CI}:-7.27$ to -2.11$)$ in favour of the intervention, measured at the end of the intervention (three trials, 146 participants, low quality evidence). This reduction appeared to be sustained at 12-24 months followup (6-12 months post intervention).

Only one very low quality trial examined the effectiveness of an energy restricted (57 participants) and dairy rich (59 participants) diets, and reported a small reduction in BMI $z$-score (MD -0.1 units [95\% CI: -0.11 to -0.09 ]), but this reduction was maintained at 36 months in only the dairy rich arm. Only one trial documented adverse events stating that none had occurred. Three trials reported healthrelated quality of life, with improvement shown in some, but not all domains, whilst behaviour change and parentchild relationships were reported inconsistently. No data were reported for all-cause mortality, morbidity, and socioeconomic effects.

A large cluster RCT $(n=475)$ was included in the review but not included in the meta-analysis due to possible methodological bias. This trial demonstrated a statistically non-significant change in BMI $z$-score (MD: -0.05 units [95\% CI: -0.14 to 0.04$]$ ) over the one year intervention (no post intervention follow-up data was provided).

\section{Interventions for school aged children under 12 years}

Lifestyle interventions targeting parents as the sole agents for change Loveman et al. (2015) [30] conducted searches up to March 2015, and identified 20 trials and ten ongoing studies. The 20 trials comprised of 3057 participants, and reported a median mean baseline age of 8 years, a median female proportion of $\sim 60 \%$, and where reported a median baseline BMI $z$-score of 2.2 in the intervention group and 2.3 in the control group. The proportion of white participants ranged from 54 to $100 \%$. All but one trial were published from 2000 onwards, and were conducted in seven countries (one upper-middle income). The sample size of individual trials ranged from 15 to 645 and the duration of the intervention ranged from 2.25 to 24 months. All but three trials included a period of post intervention follow-up ranging from 2.75 to 18.5 months, giving rise to a follow-up from baseline ranging from 5.5 to 24 months. Whilst the content of the interventions varied considerably, the comparators also differed, and so the outcomes were metaanalysed by comparator group.

BMI $z$-score was the most frequently reported outcome measure. The mean difference in BMI $z$-score at longest follow-up (10 to 24 months) was -0.04 units $(95 \%$ CI: -0.15 to 0.08 ) for three trials (267 participants) comparing parent-only intervention with parent child intervention. A similar statistically non-significant change in BMI $z$-score was seen when comparing parent-only interventions to minimal contact control interventions at the longest followup period (9 to 12 months), with a mean difference of -0.01 units (95\% CI: -0.07 to 0.09 ) (one trial, 165 participants). For the two trials (136 participants) comparing parent-only interventions with a waiting list control, a statistically significant change in BMI $z$-score was observed in favour of the intervention at the longest followup period (10 to 12 months) with a mean difference of -0.10 units (95\% CI: -0.19 to -0.01 ). Where the comparator was a concomitant intervention, no metaanalysis was reported due to a very high degree of heterogeneity $\left(I^{2}=94 \%\right)$.

Secondary outcomes reported in the summary of findings table included parent-child relationships which were assessed in three studies (low quality evidence): two demonstrated a small effect in favour of the parent-only 
intervention and one showed no effect. Whilst two studies reported that no serious adverse events occurred, generally adverse events were not reported. Data on morbidity, allcause mortality and socioeconomic effects were not reported.

Lifestyle interventions targeting the child-only, or child and parent Mead et al. (2017) [32], conducted searches up to July 2016 and identified 70 trials (8,461 participants), four of which were cluster trials, and 20 ongoing studies. Although the vast majority of trials included both the child and parent/care giver $(n=65)$ and comprised a dietary, physical activity and behavioural component $(n=49)$, the delivery and content of the interventions varied considerably. Individual trial sample sizes ranged from 16 to 686 participants, with a median mean age of 10 years at baseline (with only 15 trials including participants with a mean age of $<9$ years). The median proportion of female participants was approximately 55\% (ranging from 26 to $100 \%$ ). Where reported, the median proportion of white participants was 80 and $71 \%$ in the intervention and control arms, respectively (ranging from 0 to 100\%). Median of the mean BMI $z$-score at baseline was 2.2 (ranging from 1.3 to 5.6), the duration of the interventions ranged from 0.25-24 months, and follow-up from baseline ranged from 5.5-36 months. Just over half of the trials $(n=37)$ had a period of post intervention follow-up, with a median duration of 10 months.

The majority of trials $(n=63)$ were published from 2000 . Trials took place in 18 high-income countries and three upper-middle-income countries. Setting varied significantly across studies, although half $(n=36)$ took place in primary or secondary care. Fifteen trials evaluated an additional element as part of a concomitant intervention, and were consequently analysed separately. A further two separate analyses were also conducted for the four cluster trials and two maintenance trials. Of these separately assessed trials, two of the clusters were subject to methodological queries which precluded them from analysis, and the remaining studies did not demonstrate any substantial impact on BMI.

Twenty four trials reporting BMI could be pooled for analysis, and demonstrated a change in BMI in favour of the intervention (measured at last available point of follow-up) of $-0.53 \mathrm{~kg} / \mathrm{m}^{2}$ (95\% CI: -0.82 to -0.24 ); 2785 participants). Thirty-seven trials reported BMI $z$-score suitable for metaanalysis, which resulted in a change in favour of intervention (measured at last available point of follow-up) of -0.06 units, (95\% CI: -0.10 to -0.02 ); 4019 participants).

As the main meta-analyses revealed significant heterogeneity, subgroup analyses were conducted to examine the impact of: type of intervention, type of comparator, risk of attrition bias, setting of intervention, duration of post intervention follow-up period, parental involvement and severe obesity at baseline. None of the subgroup analyses gave rise to a consistent effect that differed significantly from the overall pooled effect for both BMI and BMI $z$ score. However, subgroup analysis of BMI by duration of post intervention follow-up period (no post intervention follow-up [15 trials] vs. follow-+up at: <6 [3 trials], 6-12 [2 trials] and $>12$ [4 trials] months) demonstrated that intervention effects only remained significant immediately post intervention. A similar pattern was also observed for BMI $z$-score, although it did not reach significance. These findings align with data from the two trials (263 participants) identified in this review that specifically examined the impact of a post intervention maintenance period on BMI $z$-score and found no significant intervention effect.

Thirty-one trials documented whether serious adverse events occurred, although the vast majority $(n=29)$ reported zero occurrence. In the two trials that reported a serious adverse event occurrence, examples included influenza, muscular-skeletal surgery or injuries, however none were considered to be related to the study. Six trials reported a range of adverse events in a small percentage of participants (examples included elevated triglycerides, blood pressure and cholesterol in both groups in one trial, and a range of accidents, infections, and skin rashes across groups, none of which were deemed to be related to the trial). Only a small number of trials reported secondary outcomes with data suitable for meta-analysis: parent reported and child reported health-related quality of life was reported in five trials (718 participants) and three trials (164 participants), respectively; two trials reported on selfesteem (144 participants); two trials (168 participants) reported change in caloric intake; and six trials (744 participants) reported accelerometry measured physical activity. However, none of the analyses demonstrated a significant difference between intervention and control. Two trials (55 participants) reported minutes per day of TV viewing, and found a small significant reduction of $6.6 \mathrm{~min}$ per day in favour of the intervention. The data on morbidity, all-cause mortality and socioeconomic effects were not reported.

\section{Interventions for children 12 years and older}

Al-Khudairy et al. (2017) [33] conducted searches up to July 2016, and 44 trials (4781 participants; median mean age at baseline: 14.3 years), and 50 ongoing studies were identified. All studies (apart from two) were published between 2000 and 2017, and were conducted in 15 countries (with five trials conducted in four upper-middleincome countries). The duration of the interventions ranged from 6 weeks to 2 years, with follow-up from baseline ranging from 24 weeks to 2 years, with a post intervention 
follow-up period (median length 6 months; range 121 months) in just over half of all studies. The setting and content of the interventions varied considerably across the trials; five trials focused solely on physical activity interventions, five on diet only interventions, and 34 on multicomponent interventions. Sample size ranged from 10 to 521 participants. Median of the mean (and range) baseline BMI and BMI $z$-score across the studies in the intervention groups were $32.4 \mathrm{~kg} / \mathrm{m}^{2}\left(26.6-45.5 \mathrm{~kg} / \mathrm{m}^{2}\right)$ and 2.2 units (1.92-4.2 units), respectively, and in the control groups $31.84 \mathrm{~kg} / \mathrm{m}^{2}\left(26.6-45.5 \mathrm{~kg} / \mathrm{m}^{2}\right)$ and 2.2 units $(1.81-4.3$ units), respectively. The median proportion of female participants was $55.8 \%$ in the intervention groups and $54.5 \%$ in the controls (ranging from 0-100\%); in the 19 trials reporting ethnicity, the proportion and range of white participants was $58.8 \%$ for the intervention groups and $34.8 \%$ for the controls (ranging from $0-100 \%$ ).

For the trials that could be pooled for meta-analysis, the overall mean difference in change in BMI at the last available measurement point was $-1.18 \mathrm{~kg} / \mathrm{m}^{2}$ (95\% CI: -1.67 to -0.69) (2774 participants; 28 trials), while the change in BMI $z$-score was -0.13 units (95\% CI: -0.21 to -0.05 ) (2399 participants; 20 trials). This reduction remained when examined in those trials with long (18-24 months) follow-up from baseline: BMI $-1.49 \mathrm{~kg} / \mathrm{m}^{2}$ (95 CI: -2.56 to -0.41$)(760$ participants; six trials) and BMI $z$-score -0.34 units $(95 \% \mathrm{CI}$ : -0.66 to -0.02 ) (602 participants; five trials). As expected intervention effects were larger when compared to no intervention or usual care, than those compared to concomitant interventions. The length of the post intervention follow-up period had no significant effects on BMI. Further subgroup analyses revealed that the type of intervention (multicomponent, physical activity only or diet only) had little effect on outcomes although the vast majority of trials were multicomponent. Similarly, parental involvement in the intervention, the intervention setting, mode, and theoretical basis of intervention did not significantly alter the overall effect estimates. Only five trials documented adverse events, three of which reported no events; one stated $6.4 \%$ of participants experienced an adverse event (but no further details provided) and only one reported the occurrence of adverse events documented as ranging from 19 to $25 \%$. Seven trials (972 participants) demonstrated an improvement in health-related quality of life at a follow-up of 6 to 24 months (SMD 0.44 (95\% CI: 0.09 to $0.79)$ ). Lifestyle related behaviours were measured too inconsistently to summarise, while measures of all-cause mortality, morbidity and socioeconomic effects were not reported.

\section{Drug interventions for the treatment of obesity in children and adolescents}

Mead et al. (2016) [29] conducted searches up to March 2016 and identified 21 trials (11 metformin [including one trial arm with Metformin and Fluoxetine], six Sibutramine, four Orlistat), and eight ongoing studies. Duration of the interventions ranged from 2.75 to 12.5 months, with duration of follow-up (from baseline) ranging from 5.5 to 23 months. It is important to note that only four trials reported post intervention follow-up. In total 2484 children (mean baseline age range 10-16 years [median 13.7 years], mean baseline BMI range $26-42 \mathrm{~kg} / \mathrm{m}^{2}$ [median $\sim 35 \mathrm{~kg} / \mathrm{m}^{2}$ ]) participated in the included trials, which were undertaken in secondary care settings and conducted in the last two decades (1999-2010). The trials took place in 12 different countries (four upper-middle income) with an individual trial sample size ranging from 24 to 539 participants, and completion rates ranging from 36 to $100 \%$ (median $78.6 \%)$.

Eighteen trials were placebo controlled, and 17 of these also included a concomitant lifestyle intervention. Ethnicity was clearly reported in 10 out of the 21 trials with the proportion of white participants ranging from 37 to $92 \%$. BMI was meta-analysed for 16 trials (1884 participants) at 6 months (14 trials) and 12 months (two trials), which was the end of the active intervention in all but one trial. A small but statistically significant mean difference in BMI was observed: $-1.3 \mathrm{~kg} / \mathrm{m}^{2}$ (95\% CI: -1.9 to -0.8 ) in favour of the intervention. When these data were analysed by drug type, Sibutramine, Metformin and Orlistat all demonstrated a reduction in BMI. Additional subgroup analyses indicated statistically significant differences, favouring studies with higher dropout and from middle-income countries. The most common adverse events were: gastrointestinal in the Orlistat and Metformin trials; and tachycardia, constipation and hypertension in the Sibutramine trials. Serious adverse events were reported in five trials (1347 participants) resulting in a relative risk of 1.43 (95\% CI: 0.63 to 3.25 ). Health-related quality of life was only reported in two trials (86 participants), with no significant between group differences seen in the trial reporting findings from the SF36 health questionnaire. One suicide was reported in an Orlistat group. Morbidity was reported in only one trial (533 participants) resulting in a small between group difference in new gallstone development in an Orlistat arm. Data on socioeconomic effects were not reported.

\section{Surgery for the treatment of obesity in children and adolescents}

Ells et al. (2015) [28] conducted searches up to March 2015, and identified one recent Australian RCT examining the effectiveness of a laparoscopic adjustable band compared to a multicomponent lifestyle intervention (usual care). Fifty predominantly female adolescent participants with severe obesity (mean age 16 years, mean BMI over 40 ), took part in the trial. Between baseline and last point of measurement 
Table 1 Outstanding research questions

What weight management interventions are most effective in low and middle-income countries?

What weight management interventions are most effective for children with disabilities, complex health needs or very severe forms of obesity?

What weight management interventions are most effective in specific ethnic, religious and culturally diverse groups?

How cost effective are child and adolescent weight management programmes?

What are the key intervention components that promote success?

What are the key family characteristics/environments that promote success?

What is the optimal role of parents within different age groups?

What behaviour change strategies are most useful?

What maintenance programmes are required to help improve the sustainability of positive weight management improvements?

What are the benefits of dual family weight management i.e. interventions that address weight management in overweight children and overweight parents simultaneously in the same intervention?

What are the impacts of emerging new technologies such as e-health in children?

What are the long-term benefits, and safety of drug interventions in children and adolescents with obesity?

How clinically effective is restrictive or mal-absorptive bariatric surgery in treating obesity in adolescents from different backgrounds?

(24 months) participants in the surgery arm experienced a significant $12.7 \mathrm{~kg} / \mathrm{m}^{2}$ (95\% CI: 11.3 to 14.2 ) reduction in BMI compared to a reduction of $1.3 \mathrm{~kg} / \mathrm{m}^{2}$ (95\% CI: 0.4 to $2.9)$ in the control arm. The surgery participants also experienced improvements in two of eight quality of life concepts, when compared to the control. Post intervention morbidity (metabolic syndrome) was reported in four patients completing the control arm and no patients in the intervention arm. No other secondary outcome data were reported. Four ongoing studies were identified which may help strengthen future evidence for surgery interventions in this population group.

\section{Discussion}

This overview provides a comprehensive update to Oude Luttikhuis, 2009 [34]. However, despite a dramatic increase in the number of trials conducted over the last eight years, overall the findings remain similar. The outcomes also align with more recent systematic reviews of parent-only interventions; [38, 39] educational interventions to treat obesity in 6- to 12-year-old children;[40] school-based interventions [41] and lifestyle interventions for children up to 18 years [42]. BMI $z$-score change by age group also followed a similar pattern to changes reported in a recent observational study [43].

All six reviews provided good quality evidence with high R-AMSTAR scores, thus providing a high degree of confidence in the review findings and clinical relevance [36]. However, the overall quality of the trials included in the reviews was low, with improvement required across most of the risk of bias domains, but in particular attrition and selective reporting. Performance bias was also an identified risk in many of the non-drug trials, reflecting the difficulties in blinding lifestyle and surgical interventions.

\section{Implications for research}

Despite a sizeable evidence base, there remain a number of important gaps (Table 1). Therefore, serious consideration should be given to ensuring all new trials follow the CONSORT criteria, use standardised outcome assessment criteria and validated measurement tools, to facilitate comparisons across trials. Trials co-ordinators should also ensure that long-term (>12 month) post intervention follow ups are conducted, and details on all adverse events and maintenance periods are clearly and consistently reported. Authors should use the TIDieR checklist [44] to provide comprehensive and reproducible trial descriptions (clearly describing both the control and intervention conditions, given the differences that can arise in usual care provision), and must ensure: (1) trials are adequately powered; (2) attrition is accounted for in an appropriate intention to treat analysis (i.e. the use of multiple imputation); (3) intervention cost is included; and (4) where possible study personnel are blinded.

Conducting more qualitative research to understand the barriers and facilitators to weight management in different populations would be advantageous, as would more process evaluations [45]. These studies may help guide implementation, tailor interventions to populations needs, and understand which approaches may work better for which populations and why.

\section{Implications for practice}

It is important to note that the vast majority of the evidence was generated in high-income countries, thus calling into question the generalisability of these findings in low- and middle-income countries. This is particularly important given the most rapid recent rises in overweight in young children from low- and lower-middle-income countries [1], and lack of cost-effectiveness data, making it difficult to effectively translate findings for lower income countries with potentially different health and political economies.

Only one trial of bariatric surgery was identified that provides insufficient evidence to assess the wider 
applicability and acceptability of this approach. A recent non-RCT study including 242 adolescents undergoing bariatric surgery at five U.S. centres reported significant improvements in weight, cardio-metabolic health, and weight-related quality of life 3 years post-surgery. However, associated risks included specific micronutrient deficiencies and the need for additional abdominal procedures [46]. A recent French review concluded that bariatric surgery is not a simple surgical intervention in teenagers, with minor side effects reported in $10-15 \%$, and severe side effects in $1-5 \%$ [47].

Drug interventions were also assessed, however, some of the trial drugs were used off license, or have been withdrawn in some countries, which coupled to the lack of longterm follow-up and safety data, makes it impossible to make any conclusive recommendations.

From the lifestyle modification reviews, the largest $(0.3$ unit) BMI $z$-score reduction was observed in the interventions targeting the youngest children ( $2-5$ years), although this was by far the smallest evidence base. However, given the tracking of excess weight into later childhood $[48,49]$ it is important to observe the effectiveness of early intervention to help prevent excess weight persisting into later childhood. Early treatment may also be important given the smallest overall reduction in BMI $z$-score (0.06 units) was observed in interventions delivered to children aged 6-11 years. This finding may reflect the challenges of intervening in this age group, who may be more influenced by the wider obesogenic environment than their younger counterparts. This age group may also be less autonomous than their older adolescent peers and may therefore rely more on parental support, yet the exact role of parents and parental weight status is not clearly described. This finding warrants further consideration given the association between parental and child obesity [50].

While any reduction in BMI $z$-score for children with overweight and obesity may be of clinical benefit, the BMI $z$-score reduction required to ameliorate any comorbidities is less clear. For example, a small observational study in young people (median age 12.4 years) with severe obesity reported that a reduction of $0.25 \mathrm{BMI} z$-score units was required to improve adiposity and metabolic health [51]. However, improvements in cholesterol were observed in children with obesity aged 7-17 years with a BMI $z$-score reduction of $<0.1$ unit [52], and improvement in insulin and cholesterol was observed in 5-19 year olds with obesity, following a BMI $z$-score reduction of 0.15 (SD 0.5) units [53]. Reduction of systemic blood pressure and arterial stiffness was also reported in pre-pubertal children with obesity following a BMI $z$-score reduction of 0.1 unit [54]. The differences in BMI $z$-score associated cardio-metabolic changes may also be affected by the use of different reference populations used to calculate the BMI $z$-score
(Farpour-Lambert personal communication). In addition to any clinical benefit, it is important to consider the public health benefits of even small BMI/BMI $z$-score reductions if feasibly achieved across an entire population [55].

As BMI it is not a direct measure of body composition, changes in fat mass may be confounded with changes in fatfree mass. This is particularly important, given the data from the UK [56], US [57] and Australia [58] demonstrates increases in central adiposity exceeding increases in BMI in children. Although other body composition measures were not the focus of this overview given variations in the use, cost and precision, each review did show (as a secondary outcome) other body composition indices were reported in the individual trials. Waist circumference was the most frequently reported measure, however, meta-analyses of this outcome were only reported in the reviews of children [32] 6-11 years (final follow-up: MD $-2.41 \mathrm{~cm}, 95 \% \mathrm{CI}-3.59$ to $-1.23 ; P<0.0001 ; 11$ trials; 1325 participants) and $12-$ 17 years [33] (final follow-up: MD $-2.26 \mathrm{~cm}, 95 \% \mathrm{CI}$ -3.80 to $-0.72 ; P=0.004 ; 17$ trials; 1997 participants), thus demonstrating a parallel reduction in waist circumference and BMI as a result of lifestyle interventions in school age children.

Although intervention content, format and delivery varied significantly both within and across the included reviews, collectively there was evidence to support the role of multicomponent interventions. There was also no clear difference in terms of outcome according to setting, which may suggest that intervention content and wider context may be more important than delivery setting. Very little data were provided on the role of the family characteristics or the wider environment. Although the inclusion of parents in both the school age (6-11 years) and adolescent (12-17 years) studies did not appear to significantly impact on the overall effect of the intervention, specifically targeting children and parents with overweight in the preschool (up to 6 years) review seemed to demonstrate a dual benefit to both children and their parents. Workniak et al. [59]. also showed that parental weight status change was an independent predictor of child weight status change in a familybased weight management study of children 8-12 years. Parents have an important role in controlling their child's food and activity environment, helping their child attend treatment sessions and implement changes. Thus, intensity of parental involvement [60] and their role as an influential role models [61], may all be important contributors to effective long-term paediatric weight management.

The sustainability of any observed reduction in BMI/ BMI $z$-score is a key consideration. Whilst effects appeared to be sustained in the adolescent and preschool aged children, data from children aged 6-11 suggests effects were not sustainable. Given obesity is a chronic relapsing disease [62] manifested in an obesity conducive environment, it is 
Table 2 Practical considerations when implementing findings in practice

Evidence from surgical and drug RCTs was too limited to make inferences about use and safety, and adverse events were a serious consideration.

Lifestyle interventions can be successful in producing small reductions in BMI $z$-score with relatively low occurrence of adverse events. However obesity is a chronic relapsing condition, therefore it may be challenging to sustain changes over the longer term, thus ongoing maintenance support will be required.

As there is currently insufficient evidence to suggest which particular setting or intervention component may be more or less beneficial, it may be useful to consider the following:

$\bigcirc$ Take a multi-component approach incorporating nutrition, physical activity and behaviour change components.

$\bigcirc$ Identify high risk groups and ensure services are accessible and appropriate for them: can reasonable adjustments be made to improve inclusivity?

Co-production: develop interventions with target populations, to tailor the intervention to local needs (e.g. language/cultural adaptations; co-morbidity management; tailored time, location and delivery style). This may also help reduce attrition and poor compliance which can be problematic.

Effective communication: how best do you reach your target population and referring staff (e.g. available networks, social media, TV, radio, print)?

A truly family-based approach - ensuring support is in place for all family members with weight concerns.

$\bigcirc$ The influence of the wider environment (food taxes, availability of healthy food and drink; and opportunities to be active), and local systems (personal resources and staff training; financial coverage of treatment)

Ensure comprehensive systems are in place to record adverse events.

The needs of complex families (i.e. families in crisis, child protection issues, severe parental or child psychiatric illness) who are unlikely to participate in trials.

Consider the scalability of any intervention, and the cost to both to the provider and participants.

Evaluate and learn from local implementation, by using standardised and validated outcome measurement tools.

perhaps unsurprising that short-term effects do not persist particularly in children who may be most influenced by their wider environment.

Data on adverse events were generally not well reported across the studies in any of the lifestyle reviews, but where reported occurrence was low. However adverse events such as effects on linear growth, injuries, eating disorders and psycho-social well-being must be considered. Bariatric surgery is a major surgical intervention, with serious potential risks for operative and perioperative complications and mortality. The restrictive or mal-absorptive nature of some forms of bariatric surgery presents an additional consideration in growing children. Psychological maturity, ability to provide informed consent, the availability of family support, and provision of ongoing post-operative lifestyle support [63] should be considered. Drug interventions are also not without adverse events, which depending on the drug prescribed, include a variety of gastrointestinal and cardiovascular conditions.

In summary, this overview provides a comprehensive update on the effectiveness of obesity treatments for children 2-18 years. However, it is essential that when translated into practice, findings are interpreted within the context of local political and health systems, and population needs (Table 2). It is also important to acknowledge the limitations of RCT evidence when evaluating complex interventions. It may therefore be important to consider additional observational studies, where gaps remain in the RCT evidence.

\section{Disclaimer}

The authors alone are responsible for the views expressed in this publication and they do not necessarily represent the official position, decisions, policy or views of the World Health Organization.

Acknowledgements We would like to thank Teesside University, University of Warwick and the World Health Organization for supporting this work. We would also like to thank Claire O'Malley and Joan Olajide for kindly providing a second data and quality assessment for the drug and surgery reviews, respectively, and Alena Matzke for assistance in translating a German publication for the adolescent review.

Funding This research was funded by the Department of Nutrition for Health and Development, World Health Organization - contract number: 201538776. Karen Rees's contribution was also kindly funded by the University of Warwick Impact Fund.

\section{Compliance with ethical standards}

Conflict of interest LJE and EM are co-authors on all six reviewed publications, KR, LA-K, and LB are co-authors on four of the reviewed publications, EL and LA are co-authors on three of the reviewed publications and $\mathrm{TB}$ and $\mathrm{AD}$ are co-authors on one of the reviewed publications. LJE is seconded to Public Health England as a specialist obesity advisor 2 days a week, however, this work was undertaken within her role as Reader at Teesside University. PR-S and $\mathrm{AD}$ are full-time staff members of the World Health Organization. The remaining authors declare that they have no conflicts of interest.

Open Access This article is licensed under a Creative Commons Attribution 4.0 International License, which permits use, sharing, adaptation, distribution and reproduction in any medium or format, as long as you give appropriate credit to the original author(s) and the source, provide a link to the Creative Commons license, and indicate if changes were made. The images or other third party material in this article are included in the article's Creative Commons license, unless indicated otherwise in a credit line to the material. If material is not included in the article's Creative Commons license and your intended use is not permitted by statutory regulation or exceeds the permitted use, you will need to obtain permission directly from the copyright holder. To view a copy of this license, visit http://creativecommons. org/licenses/by/4.0/. 


\section{References}

1. WHO. Levels and trends in child malnutrition. UNICEF/WHO/ World Bank Group joint malnutrition estimates. Key findings of the 2017 edition. New York/Geneva/Washington DC: The United Nations Children's Fund, the World Health Organization and the World Bank Group; 2017.

2. WHO. Facts and figures on childhood obesity 2016. http://www. who.int/end-childhood-obesity/facts/en/. Accessed 10 Feb 2017.

3. Lobstein T. J-LR. Planning for the worst: estimates of obesity and comorbidities in school age children in 2015. Pediatr Obes. 2016;11:321-5.

4. Knai C, Lobstein T, Darmon N, Rutter H, McKee M. Socioeconomic patterning of childhood overweight status in Europe. Int J Environ Res Public Health. 2012;9:1472-89.

5. Shrewsbury V, Wardle J. Socioeconomic status and adiposity in childhood: a systematic review of cross-sectional studies 19902005. Obesity. 2008;16:275-84.

6. Wang Y, Lim H. The global childhood obesity epidemic and the association between socio-economic status and childhood obesity. Int Rev Psychiatry. 2012;24:176-88.

7. Health and Social Care Information Centre. National Child Measurement Programme - England, 2012-13 school year. 2013 https://files.digital.nhs.uk/publication/j/n/nati-chil-meas-prog-eng2016-2017-rep.pdf Accessed 05/10/2018.

8. Skinner AC, Skelton JA. Prevalence and trends in obesity and severe obesity among children in the United States, 1999-2012. JAMA Pediatr. 2014;168:561-6.

9. Hardy LL, King L, Hector D, Baur LA. Socio-cultural differences in Australian primary school children's weight and weight-related behaviours. J Paediatr Child Health. 2013;49:641-8.

10. Rajput N, Tuohy P, Mishra S, Smith A, Taylor B. Overweight and obesity in 4-5-year-old children in New Zealand: results from the first 4 years (2009-2012) of the B4School Check programme. J Paediatr Child Health. 2015;51:334-43.

11. Paulis WD, Silva S, Koes BW, van Middelkoop M. Overweight and obesity are associated with musculoskeletal complaints as early as childhood: a systematic review. Obes Res. 2014;15:5267.

12. Friedemann C, Heneghan C, Mahtani K, Thompson M, Perera R, Ward AM. Cardiovascular disease risk in healthy children and its association with body mass index: systematic review and metaanalysis. BMJ. 2012;345:e4759.

13. Narang I, Mathew JL. Childhood obesity and obstructive sleep apnea. J Nutr Metab. 2012;134202.

14. Egan K, Ettinger A. Childhood body mass index and subsequent physician-diagnosed asthma: a systematic review and metaanalysis of prospective cohort studies. BMC Pediatr. 2013;13: 121

15. Feldstein AE, Charatcharoenwitthaya P, Treeprasertsuk S, Benson JT, Enders FB, Angulo P. The natural history of nonalcoholic fatty liver disease in children: a follow-up study for up to 20 years. Gut. 2009;58:1538-44.

16. Rees R, Oliver K, Woodman J, Thomas J. The views of young children in the UK about obesity, body size, shape and weight: a systematic review. BMC Public Health. 2011;11:188.

17. Griffiths LJ, Parsons TJ, Hill AJ. Self-esteem and quality of life in obese children and adolescents: a systematic review. Int J Pediatr Obes. 2010;5:282-304.

18. Singh AS, Mulder C, Twisk JW, van Mechelen W, Chinapaw MJ. Tracking of childhood overweight into adulthood: a systematic review of the literature. Obes Rev. 2008;9:474-88.

19. Simmonds M, Llewellyn A, Owen CG, Woolacott N. Predicting adult obesity from childhood obesity: a systematic review and meta-analysis. Obes Rev. 2016;17:95-107.
20. Reilly JJ, Kelly J. Long-term impact of overweight and obesity in childhood and adolescence on morbidity and premature mortality in adulthood: systematic review. Int J Obes. 2011;35:891-8.

21. Llewellyn A, Simmonds M, Owen CG, Woolacott N. Childhood obesity as a predictor of morbidity in adulthood: a systematic review and meta-analysis. Obes Rev. 2016;17:56-67.

22. Golan M, Crow S. Targeting parents exclusively in the treatment of childhood obesity: long-term results. Obes Res. 2004;12:35761.

23. Brei MN. Current guidelines for weight loss surgery in adolescents: a review of the literature. J Pediatr Health Care. 2014;28:288-94. S. M.

24. Sherafat-Kazemzadeh R, Yanovski SZ, Yanovski JA. Pharmacotherapy for childhood obesity: present and future prospects. Int J Obes. 2013;37:1-15.

25. Buck M. Use of metformin in pediatric patients. Pediatr Pharm. 2004;10.

26. Hsia Y, Dawoud D, Sutcliffe AG, Viner RM, Kinra S, Wong IC. Unlicensed use of metformin in children and adolescents in the UK. Br J Clin Pharmacol. 2012;73:135-9.

27. Petkar R, Wright N. Pharmacological management of obese child. Arch Dis Child Educ Pract Ed. 2013;98:108-12.

28. Ells LJ, Mead E, Atkinson G, Corpeleijn E, Roberts K, Viner R, et al. Surgery for the treatment of obesity in children and adolescents. Cochrane Database Syst Rev. 2015:Cd011740.

29. Mead E, Atkinson G, Richter B, Metzendorf MI, Baur L, Finer N, et al. Drug interventions for the treatment of obesity in children and adolescents. Cochrane Database Syst Rev. 2016;11: Cd012436.

30. Loveman E, Al-Khudairy L, Johnson RE, Robertson W, Colquitt JL, Mead EL, et al. Parent-only interventions for childhood overweight or obesity in children aged 5 to 11 years. Cochrane Database Syst Rev. 2015:Cd012008.

31. Colquitt JL, Loveman E, O'Malley C, Azevedo LB, Mead E, AlKhudairy L, et al. Diet, physical activity, and behavioural interventions for the treatment of overweight or obesity in preschool children up to the age of 6 years. Cochrane Database Syst Rev. 2016;3:Cd012105.

32. Mead E, Brown T, Rees K, Azevedo L, Whittaker V, Jones D, et al. Diet, physical activity and behavioural interventions for the treatment of overweight or obesity in school children from the age of 6 to 11 years. Cochrane Database of Syst Rev. 2017;6: CD012651.

33. Al-Khudairy L, Loveman E, Colquitt J, Mead E, Johnson R, Fraser H, et al. Diet, physical activity and behavioural interventions for the treatment of overweight or obesity in adolescents aged 12 to 17 years. Cochrane Database Syst Rev. 2017;6: CD012691.

34. Oude Luttikhuis H, Baur L, Jansen H, Shrewsbury VA, O’Malley $\mathrm{C}$, Stolk RP, et al. Interventions for treating obesity in children. Cochrane Database Syst Rev. 2009:CD001872.

35. Becker L, Oxman A Chapter 22: overviews of reviews. In: Higgins J, Green S, editors. Cochrane handbook for systematic reviews of interventions version 510 (2011). The Cochrane Collaboration. www.cochrane-handbook.org. https://training.cochra ne.org/handbook accessed 05/10/2018

36. Kung J, Chiappelli F, Cajulis OO, Avezova R, Kossan G, Chew L, et al. From systematic reviews to clinical recommendations for evidence-based health care: validation of revised assessment of multiple systematic reviews (R-AMSTAR) for grading of clinical relevance. Open Dent J. 2010;4:84-91.

37. Higgins J. Assessing risk of bias in included studies. In: Higgins J, Green S, editors. Cochrane Handbook for Systematic Reviews of Interventions Version 510 [updated March 2011]: Chapter 8: The Cochrane Collaboration. Available from www.cochrane-ha 
ndbook.org. https://training.cochrane.org/handbook accessed 05/ $10 / 2018$

38. Jull A, Chen R. Parent-only vs. parent-child (family-focused) approaches for weight loss in obese and overweight children: a systematic review and meta-analysis. Obes Rev. 2013;14:761-8.

39. Ewald H, Kirby J, Rees K, Robertson W. Parent-only interventions in the treatment of childhood obesity: a systematic review of randomized controlled trials. J Public Health. 2014;36:476-89.

40. Sbruzzi G, Eibel B, Barbiero SM, Petkowicz RO, Ribeiro RA, Cesa CC, et al. Educational interventions in childhood obesity: a systematic review with meta-analysis of randomized clinical trials. Prev Med. 2013;56:254-64.

41. Amini M, Djazayery A, Majdzadeh R, Taghdisi MH, Jazayeri S. Effect of school-based interventions to control childhood obesity: a review of reviews. Int J Prev Med. 2015;6:68.

42. Ho M, Garnett SP, Baur L, Burrows T, Stewart L, Neve M, et al. Effectiveness of lifestyle interventions in child obesity: systematic review with meta-analysis. Pediatrics. 2012;130:e1647-71.

43. Maggio AB, Saunders Gasser C, Gal-Duding C, Beghetti M, Martin XE, Farpour-Lambert NJ, et al. BMI changes in children and adolescents attending a specialized childhood obesity center: a cohort study. BMC Pediatr. 2013;13:216.

44. Hoffmann TC, Glasziou PP, Boutron I, Milne R, Perera R, Moher $\mathrm{D}$, et al. Better reporting of interventions: template for intervention description and replication (TIDieR) checklist and guide. BMJ. 2014;348:g1687.

45. Moore GF, Audrey S, Barker M, Bond L, Bonell C, Hardeman W, et al. Process evaluation of complex interventions: Medical Research Council guidance. BMJ. 2015;350:h1258.

46. Inge TH, Courcoulas AP, Jenkins TM, Michalsky MP, Helmrath MA, Brandt ML, et al. Weight loss and health status 3 years after bariatric surgery in adolescents. $N$ Engl J Med. 2016;374: 113-23.

47. Coutant R, Bouhours-Nouet N, Donzeau A, Fauchard M, Decrequy A, Malka J, et al. Bariatric surgery in adolescents with severe obesity: Review and state of the art in France. Ann Endocrinol (Paris). 2017;78:462-8.

48. Mead E, Batterham AM, Atkinson G, Ells LJ. Predicting future weight status from measurements made in early childhood: a novel longitudinal approach applied to Millennium Cohort Study data. Nutr Diabetes. 2016;6:e200.

49. Public Health England. Changes in the weight status of children between the first and final years of primary school. A longitudinal analysis of data from the National Child Measurement Programme in four local authorities in England between 2006/07 and 2014/15. London: Wellington House, Public Health England; 2017.

50. Lake JK, Power C, Cole TJ. Child to adult body mass index in the 1958 British birth cohort: associations with parental obesity. Arch Dis Child. 1997;77:376-81.
51. Ford AL, Hunt LP, Cooper A, Shield JP. What reduction in BMI SDS is required in obese adolescents to improve body composition and cardiometabolic health? Arch Dis Child. 2010;95:25661.

52. Kolsgaard ML, Joner G, Brunborg C, Anderssen SA, Tonstad S, Andersen LF. Reduction in BMI z-score and improvement in cardiometabolic risk factors in obese children and adolescents. The Oslo Adiposity Intervention Study - a hospital/public health nurse combined treatment. BMC Pediatr. 2011;11:47.

53. Kirk S, Zeller M, Claytor R, Santangelo M, Khoury PR, Daniels SR. The relationship of health outcomes to improvement in BMI in children and adolescents. Obes Res. 2005;13:876-82.

54. Farpour-Lambert NJ, Aggoun Y, Marchand LM, Martin XE, Herrmann FR, Beghetti M. Physical activity reduces systemic blood pressure and improves early markers of atherosclerosis in pre-pubertal obese children. J Am Coll Cardiol. 2009;54:2396406.

55. Rose G. Sick individuals and sick populations. Int J Epidemiol. 2001;30:427-32.

56. Griffiths C, Gately P, Marchant PR, Cooke CB. A five year longitudingal study investigating the prevalence of childhood obesity: comparison of BMI and waist circumference. Public Health. 2013;127:1090-6.

57. Freedman DS, Kit BK, Ford ES. Are the recent secular increases in waist circumference among children and adolescents independent of changes in BMI? PLoS ONE. 2015;10:e0141056.

58. Garnett SP, Baur L, Cowell CT. The prevalence of increased central adiposity in Australian schoo children 1985 to 2007. Obes Rev. 2011;12:887-96.

59. Wrotniak BH, Epstein LH, Paluch RA, Roemmich JN. Parent weight change as a predictor of child weight change in familybased behavioral obesity treatment. Arch Pediatr Adolesc Med. 2004; $158: 342-7$.

60. van der Kruk JJ, Kortekaas F, Lucas C, Jager-Wittenaar H. Obesity: a systematic review on parental involvement in long-term European childhood weight control interventions with a nutritional focus. Obes Rev. 2013;14:745-60.

61. Zarychta K, Mullan B, It AL. Doesn't matter what they say, it matters how they behave: parental influences and changes in body mass among overweight and obese adolescents. Appetite. 2016;96:47-55.

62. Bray GA, Kim KK, Wilding JPH.on behalf of the World Obesity Federation. Obesity: a chronic relapsing progressive disease process. a position statement of the World Obesity Federation. Obes Rev. 2017;18:715-23.

63. Zalesin KC, Franklin BA, Miller WM, Nori Janosz KE, Veri S, Odom J. et al.Preventing weight regain after bariatric surgery: an overview of lifestyle and psychosocial modulators.Am J Lifestyle Med. 2009;4:113-20. 